\title{
Tunka-133: the New EAS Cherenkov Light Array for Cosmic Ray Study $\left(10^{15}-10^{18} \mathrm{eV}\right)$
}

B.V.Antokhonov ${ }^{a}$, S.F.Berezhnev ${ }^{a}$, D.Besson ${ }^{g}$, N.M.Budnev $^{b}$, A. Chiavassa $^{e}$, O.A. Chvalaiev ${ }^{b}$, O.A. Gress ${ }^{b}$, A.N.Dyachok ${ }^{b}$, N.I.Karpov ${ }^{a}$, N.N.Kalmykov ${ }^{a}$, E.N.Konstantinov ${ }^{b}$, A.V.Korobchenko ${ }^{b}$, E.E. Korosteleva ${ }^{a}$, V.A.Kozhin ${ }^{a}$, L.A.Kuzmichev ${ }^{* a}$, B.K. Lubsandorzhiev ${ }^{c}$, R.R. Mirgazov ${ }^{b}$, M.I. Panasyuk ${ }^{a}$, L.V.Pankov ${ }^{b}$, E.G. Popova ${ }^{a}$, V.V. Prosin ${ }^{a}$, V.S. Ptuskin ${ }^{d}$, Yu.A. Semeney ${ }^{b}$, B.A.Shaibonov ${ }^{c}$, A.A.Silaev ${ }^{a}$, A.A. Silaev (junior) ${ }^{a}$, A.V. Skurikhin ${ }^{a}$, J.Snyder ${ }^{g}$, C.Spiering ${ }^{f}$, M.Stockham ${ }^{g}$, L.G.Sveshnikova ${ }^{a}$, R.Wischnewski ${ }^{f}$, I.V.Yashin ${ }^{a}$, A.V.Zablotsky ${ }^{a}$, A.V. Zagorodnikov ${ }^{b}$

${ }^{a}$ Skobeltsyn Institute of Nuclear Physics, Moscow State University, Russia

${ }^{b}$ Institute of Applied Physics, Irkutsk State University, Irkutsk, Russia

${ }^{c}$ Institute of Nuclear Research, Russian Academy of Sciences, Moscow, Russia

${ }^{d}$ Institute of Terrestrial Magnetism, Ionosphere and Radio Wave Propagation, Russian Academy of Sciences, Moscow,Russia

'Dipartimento di Fisica Generale dell'Universita' and INFN, Torino, Italy

${ }^{f}$ DESY, Zeuthen, Germany

${ }^{g}$ Dept. of Physics and Astronomy, University of Kansas, USA

A new EAS Cherenkov light array Tunka-133 with about $1 \mathrm{~km}^{2}$ geometric area has been installed in the Tunka Valley (50 km from Lake Baikal). The array will allow a detailed study of the cosmic ray energy spectrum and mass composition in the energy range of $10^{15}-10^{18} \mathrm{eV}$. This energy range is of crucial importance for understanding of the origin and propagation of cosmic rays in the Galaxy. The completed Tunka-133 array has been taking data since October 2009. We give an overview on the array construction and DAQ, preliminary results from data collected during the first winter run and plans for a significant detector upgrade, including an increase of the effective area and the deployment of radio-antennas and muon detectors.

25th Texas Symposium on Relativistic Astrophysics - TEXAS 2010

December 06-10, 2010

Heidelberg, Germany

* Speaker.

${ }^{\dagger}$ This work is supported by Russian Federation Ministry of Science and Education (G/K P681,P1242, RNP 2.2.1.1/1483, RNP 2.1.1/1539), the Russian Foundation for Basic Research (grants 10-02-00222, 10-02-10001, 0902-12287-ofi-m). Correspondence to kuz@dec1.sinp.msu.ru 


\section{Introduction}

The study of primary energy spectrum and mass composition in the energy range $10^{15}-10^{18} \mathrm{eV}$ is of crucial importance for the understanding of the origin of cosmic rays and of their propagation in the Galaxy.

The change from light to heavier composition with growing energy marks the energy limit of cosmic ray acceleration in galactic sources ( $\mathrm{SN}$ remnants), and of the galactic containment. This effect was described by theoretical calculations ([1], [2], [3] ) and confirmed by several experiments (e.g. [4], [5], [6]). An opposite change from heavy to light composition at higher energy would testify the transition from galactic to extragalactic sources. An experimental evidence of such behavior of the composition was reported in [7], [8].

To measure the primary cosmic ray energy spectrum and mass composition in the above mentioned intermediate energy range, the new array Tunka-133 ([9], [10]), with nearly $1 \mathrm{~km}^{2}$ internal area has been deployed in the Tunka Valley, Siberia. It records EAS Cherenkov light using the atmosphere of the Earth as a huge calorimeter and has much better energy resolution $(\sim 15 \%)$ than EAS arrays detecting only charged particles.

\section{The Tunka-133 Array}

The Tunka-133 array consists of 133 optical detectors based on PMT EMI 9350 with a hemispherical photocathode of $20 \mathrm{~cm}$ diameter. The detectors are grouped into 19 clusters, each cluster with seven detectors - six hexagonally arranged detectors and one in the center. The distance between the detectors in the cluster is $85 \mathrm{~m}$ (see Fig.11).

An optical detector consists of a $50 \mathrm{~cm}$ diameter metallic cylinder, containing a PMT. The container window is directed to zenith and covered with plexiglass plate heated against hoar-frost and dew. The detector is equipped with a remotely controlled lid protecting the PMT from sunlight and precipitation. The detection efficency of the opical module decreases smoothly to $\sim 80 \%$ of that at the vertical efficiency at $35^{\circ}$ an to $\sim 50 \%$ at $45^{\circ}$ [11].

The Cherenkov light pulses are sent via $95 \mathrm{~m}$ coaxial cable RG58 to the center of each cluster and digitized. The minimum pulse width is about $20 \mathrm{~ns}$ (FWHM). The dynamic range of the amplitude measurement is about $3 \cdot 10^{4}$. This is achieved by means of two channels for each detector extracting the signals from the anode and from an intermediate dynode of the PMT with different additional amplification factors.

The array DAQ system is arranged in a hierarchical way from the cluster electronic module to the central DAQ station. Each cluster electonics is connected to the DAQ center with a multi-wire cable consisting of four copper wires and four optical fibers.

The cluster electronics includes the cluster controller, 4 four-channel FADC boards, an adapter unit for connection with optical modules and a special temperature controller. The 12 bit and $200 \mathrm{MHz}$ sampling FADC boards are based on AD9430 fast ADCs and FPGA XILINX Spartan XC3S300 microchips. The cluster controller consists of an optical transceiver, a synchonization module, a local time clock and a trigger module. The optical transceiver operating at $1000 \mathrm{MHz}$ is responsible for data transmission and formation of $100 \mathrm{MHz}$ synchronization signal for cluster clocks. The cluster trigger (the local trigger) is formed by the coincidence of at last three pulses 
from optical detectors exceeding the threshold within a time window $0.5 \mu \mathrm{s}$. The time mark of the local trigger is fixed by the cluster clock. The accuracy of the time synchronization between different clusters is about $10 \mathrm{~ns}$.

The central DAQ station cosists of 4 DAQ boards strongly synchronized by a single $100 \mathrm{MHz}$ oscillator. The boards are connected to the master PC by $100 \mathrm{MHz}$ Ethernet lines.

\section{Data processing and reconstruction of EAS parameters}

The primary data record for each Cherenkov light detector contains 1024 amplitude values in steps of $5 \mathrm{~ns}$. Thus, the waveform of every pulse is recorded, together with the preceding noise, as a total trace of $5 \mu \mathrm{s}$ wide. To derive the three main parameters of the pulse: front delay at a level 0.25 of the maximum amplitude $t_{i}$, pulse area $Q_{i}$ and full width at half-maximum (FWHM) $\tau_{i}$, the method of pulse fitting with a smooth curve is used. The waveform of EAS Cherenkov light pulse is rather complicated and can't be fitted with a simple function as e.g. a Gauss or gamma function. Therefore, a function was constructed which separately approximates front and tail of the pulse [12].

The reconstruction of the EAS core position is performed with two methods - one by fitting the measured charges $Q_{i}$ with the lateral distribution function (LDF) and one by a new method of fitting the measured pulse widths $\tau_{i}$ by the width-distance function (WDF).

The LDF shape is described by an expression with a single parameter, the steepness $\mathrm{P}$ [13]. $\mathrm{P}$ is strictly connected with the distance from the array to the EAS maximum [11]. This connection is used to measure the shower maximum depth $X_{\max }$.

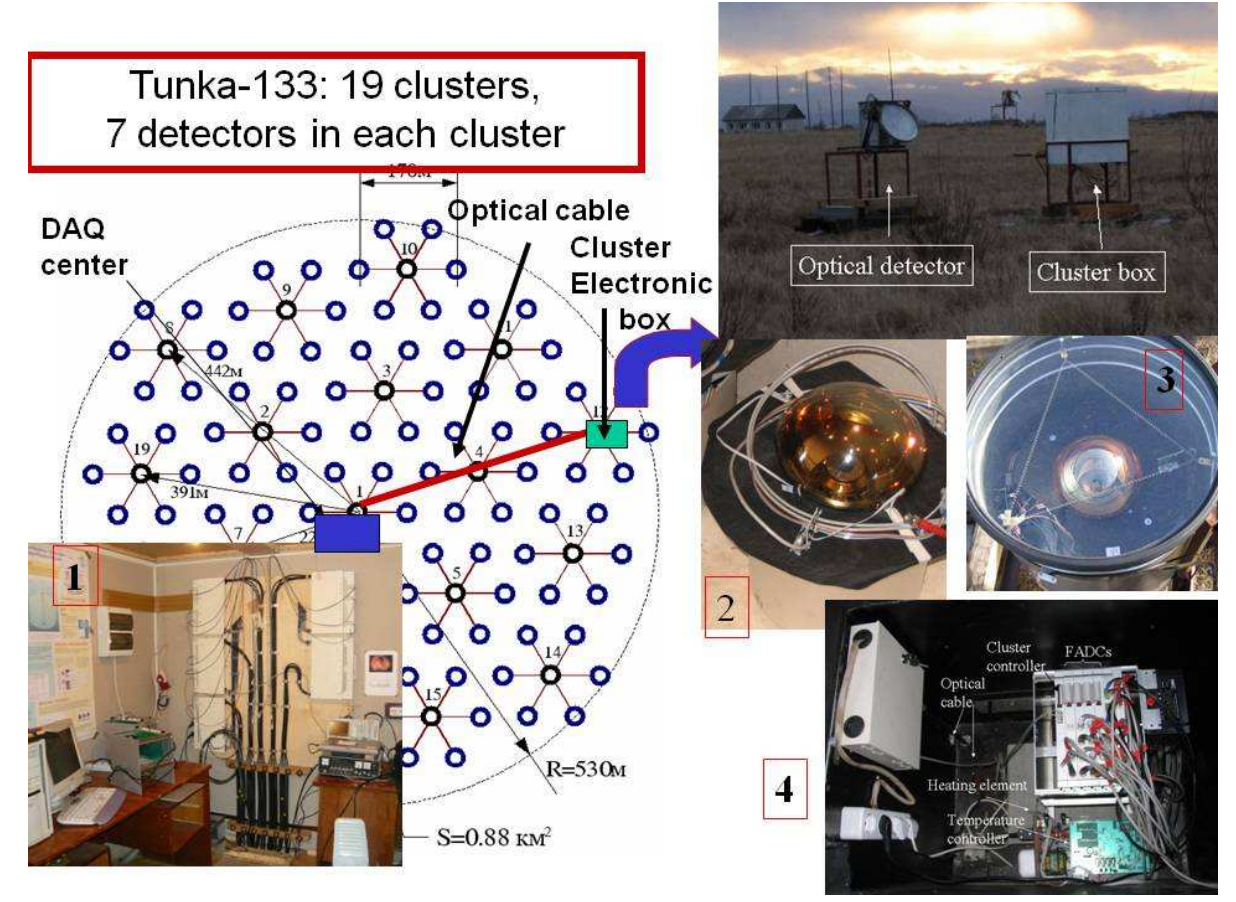

Figure 1: Tunka-133: 1. Controll room. 2. PMT insude suppoter cylinder. 3 . Up-side view of the optical decector. 4. Cluster electronics. 
The shape of the WDF is obtained from CORSIKA simulations. We used 270 events simulated for both primary protons and iron nuclei, with energies from 10 to $30 \mathrm{PeV}$ and zenith angles from $0^{\circ}$ to $45^{\circ}$. Details of the simulation are presented in [12]. The expression for the $\tau(R)$ dependence is presented in [11] $(\tau(R)$ - pulse width (FWHM) at the core distance $R)$. The apparatus distortion of pulses by PMT, preamplifier and the coaxial cable were taking into account in this expression. Pulse width $\tau$ at fixed core distance depends on the thickness between the registration level $\left(X_{0}\right)$ and the shower maximum depth $\Delta X\left(\Delta X=X_{0} / \cos \theta-X_{\max }\right)$, see figs. 目 and 3 .

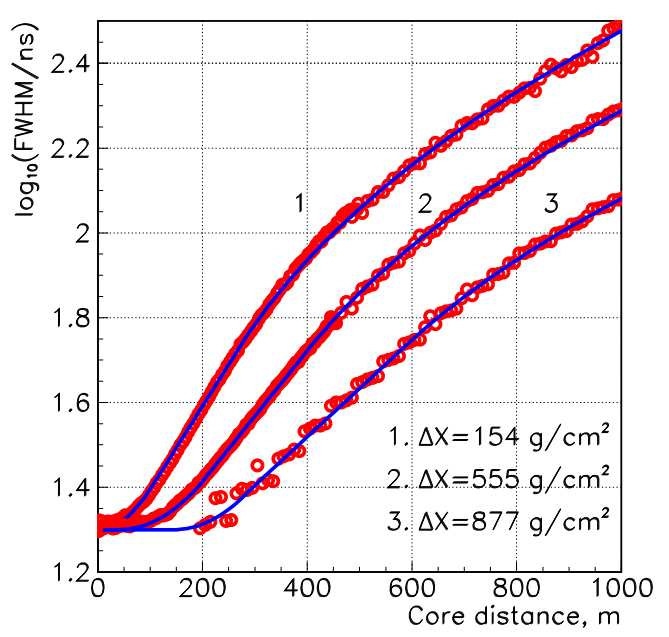

Figure 2: Dependence of pulse width $\tau$ on the core distance for showers with different relative $X_{\max }$ positions $\left(1 . \Delta X=154 \mathrm{~g} / \mathrm{cm}^{2} ; 2 . \Delta X=\right.$ $\left.555 \mathrm{~g} / \mathrm{cm}^{2} ; 3 . \Delta X=877 \mathrm{~g} / \mathrm{cm}^{2}\right)$.

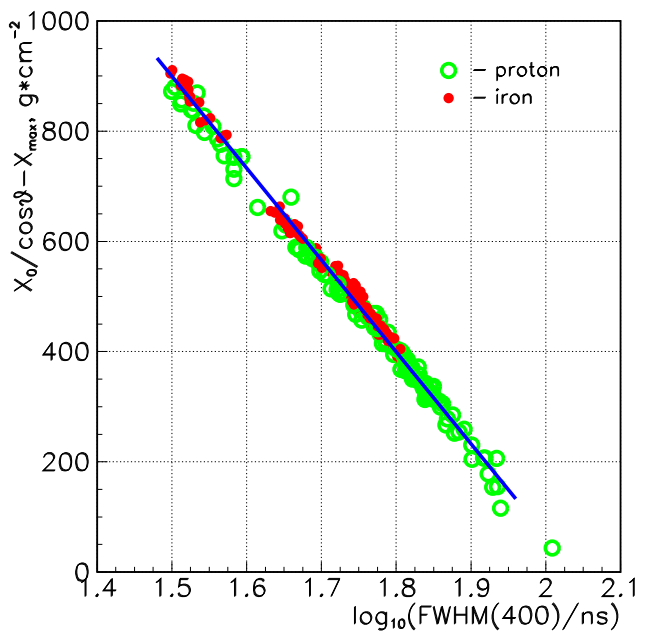

Figure 3: Thickness of matter between the array and the EAS maximum depth vs. pulse width $\tau(400)$.

\section{First results}

During the first Tunka-133 run period (winter 2009/10 with 286 hours of clean weather moonless nights), $\sim 2 \cdot 10^{6}$ events with $\mathrm{E}>10^{15} \mathrm{eV}$ have been collected. Events with core positions inside a radius of $450 \mathrm{~m}$ from the array center and with zenith angles less than $45^{\circ}$ were selected to construct the energy spectrum. Among them are about 20000 events with $E_{0}>10^{16} \mathrm{eV}$ and about 200 events with $E_{0}>10^{17} \mathrm{eV}$. An example of a real event registrated with Tunka-133 is shown in figs. 7 and 5. The energy of the event is $E=3.9 \cdot 10^{17} \mathrm{eV}$, the maximum depth is $X_{\max }=605 \mathrm{~g} / \mathrm{cm}^{2}$ It is seen that the experimental data are fit rather well with the chosen LDF and WDF functions.

The preliminary differential primary energy spectrum is presented in fig.6. We find a rather good agreement between results from Tunka-133 and its predecessor Tunka-25 [14] (shown here for $\mathrm{E} \leq 10^{16} \mathrm{eV}$ ). The energy spectrum beyond the knee looks rather complicated. First, one can see that the spectrum cannot be fit by a single power law over a wide energy range. The power law index changes from $\sim 3.2$ to $\sim 3.0$ at $\sim 2 \cdot 10^{16} \mathrm{eV}$. This fact is in good agreement with recent results of KASCADE-Grande [15]. Secondly, the spectrum has a peculiarity wich looks like a 


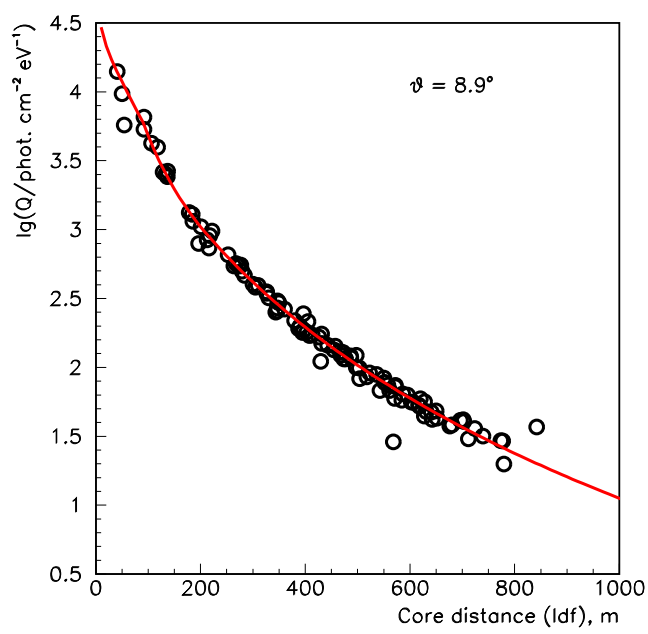

Figure 4: Tunka-133 event: Lateral distribution of EAS Cherenkov light resulting from fitting the measured light fluxes (points) with the expression (1) (curve)

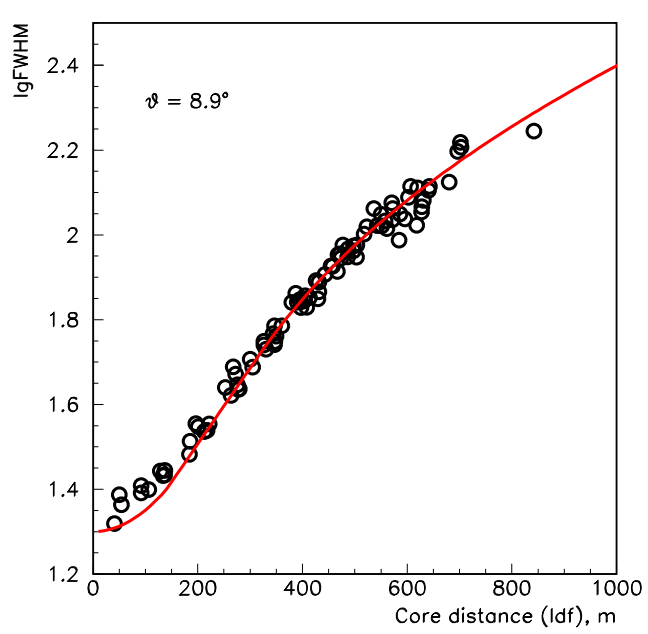

Figure 5: Tunka-133 event: Dependence of Cherenkov light pulse width on the core distance. Points - experiment, line - WDF.

"bump" in the energy range $0.8 \cdot 10^{17} \mathrm{eV}-10^{17} \mathrm{eV}$ (with 353 events in both bins, this cannot easily be explained by a statistical fluctuation). It should be noted, that the EAS experiment GAMMA reported evidence for a feature in the all-particle spectrum at comparable energy [16], as shown in fig.77.

\section{Plan for Tunka-133 upgrade}

\subsection{Deployment of six distant clusters $1 \mathrm{~km}$ away from the array center}

It seems that for small random fluctuations of the signal width, the use of WDF instead of LDF will allow a good reconstruction of events with their core position outside the geometric area of the array. In this case $X_{\max }$ can be measured via $\tau(400)$ and the energy of the shower by the density of Cherenkov light at the core distances of 400-600 m. To increase the accuracy of the core reconstruction for such events, we plan to install six new clusters at $1 \mathrm{~km}$ radius around the center of Tunka-133. These additional 42 optical detectors will increase the effective area at $10^{17} \mathrm{eV}$ by a factor of 4 . The first distant cluster was deployed in autumn 2010, the next five will be deployed during summer-autumn 2011.

\subsection{Registration of radio signal from EAS}

Like the Cherenkov light, the radio signal emitted by air showers has a calorimetric character. It has the potential to give information about the energy and the arrival direction of primary particles with very high accuracy. In addition, the form of the signal pulse and the structure of the arriving wave front have sensitivity to the depth of the shower maximum, i.e. to the mass composition [17]. To explore this method and to correlate it with the measurements of the optical method, we plan 


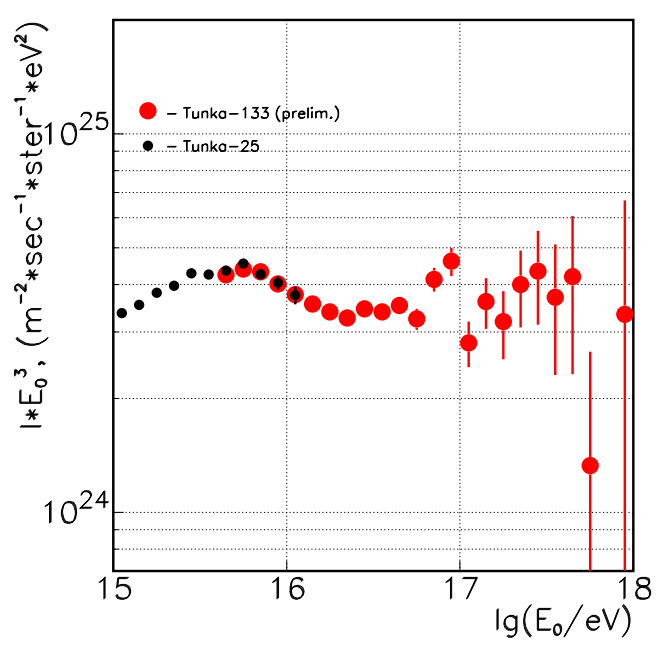

Figure 6: All-particle energy spectrum: small black points - Tunka-25 results, large red points - Tunka-133 (preliminary).

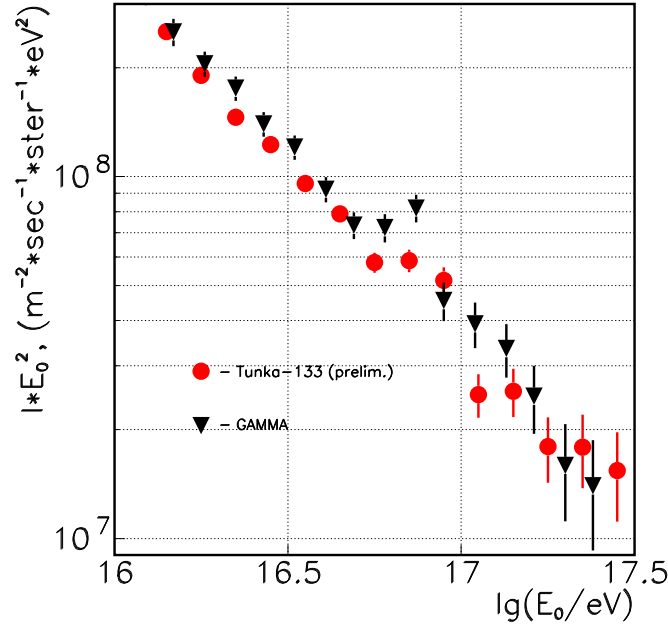

Figure 7: Comparison of all-particle energy spectra from Tunka-133 (preliminary) and GAMMA [16.

to install a net of 30-40 radio antennas for joint operation with the Cherenkov array. The Tunka site shows a rather low radio background in the interval from 40 to $80 \mathrm{MHz}$ which is a typical frequency range for radio air shower experiments. The first 2 high-gain-log periodic antennas were installed in July 2008 [10]. Two antennas of another type, the so called SALLA antenna [18] was installed in 2009 - 2010. After operation of array in the winter season 2010-2011 it will be decided which type of antenna should be chosen for the whole array.

\subsection{Registration of muons}

The deployment of scintillation counters within the Tunka array provides a cross-calibration of different methods of air shower measurements since all shower components will be recorded simultaneously. The Cherenkov light provides the energy of the primary particle and the depth of the shower maximum. The scintillation counters, buried under a layer of $1.5-2 \mathrm{~m}$ of ground, give an estimate of the number of muons. The result is of a higher precision in terms of mass composition at $10^{17}-10^{18} \mathrm{eV}$. Monte Carlo simulations with the AIRES code showed ([19]) that the measurement of the muon number with an accuracy of 5-10\%, together with the accuracy in energy and $\mathrm{X}_{\max }$ measurements achieved with Tunka-133, allow to distinguish showers produced by heavy (Fe, $\mathrm{Si}$ ) and light (proton, $\mathrm{He}$ ) nuclei. This is a key information to identify the transition from the galactic to extragalactic cosmic rays. The mentioned accuracy for the muon number requires a spacing of the muon detectors not larger than 120-150 m. It was shown in [20], that with 20 muon detectors of $10 \mathrm{~m}^{2}$ area each arranged within the Tunka-133 geometric area, an accuracy of $\sim 10 \%$ in the total muon number for energy $>10^{17} \mathrm{eV}$ can be reached. The first muon detector were installed in autumn 2010. The detector can operate independently or triggered by Tunka- 133 . The other 19 detectors will be installed in 2011 and 2012. 


\section{Conclusion}

After the first Tunka-133 operation season we present a preliminary all-particle cosmic ray spectrum between $6 \cdot 10^{15} \mathrm{eV}$ and $10^{18} \mathrm{eV}$. We conclude that the all particle energy spectrum between $10^{16} \mathrm{eV}$ and $10^{17} \mathrm{eV}$ cannot be fit with a single power law. The index $\gamma$ changes from 3.2 to 3.0 at $2 \cdot 10^{16} \mathrm{eV}$. Our energy spectrum is in good agreement for the energy range $10^{16} \mathrm{eV}$ to $6 \cdot 10^{16} \mathrm{eV}$ with the recent results of the KASCADE-Grande experiment.

In the presented preliminary energy spectrum a peculiarity is observed at energy range $0.8 \cdot 10^{17}$ - $10^{17} \mathrm{eV}$, which looks like a "bump". It seems it is incompatible with a mere statistical fluctuation. At similar energies, the GAMMA experiment reported earlier a feature in the all-particle spectrum.

The number of events above $10^{17} \mathrm{eV}$ after the first season of array operation is almost 200 . After the foreseen upgrade in summer 2011 (installation of distant clusters), the effective area of the array will increase by a factor of 4 for this energy range.

\section{References}

[1] E.G.Berezhko, H.J.Volk, Astrophys.J. 2007, 661: L175-L178; arXiv: 0704.1715.

[2] R.Aloio,V.Berezinsky et al., Phys.Rev., 2008, D77:025007; arXiv:0708.2834

[3] V.S.Ptuskin et al., Astrophys. J., 2010, 718:31-36; arXiv: 1006.0034

[4] T.Antoni et al. (KASCADE Collaboration), Astroparticle Physics 24 (2005) 1

[5] V.V.Prosin et al. (Tunka Collaboration), Nuclear Physics B (Proc. Supp.), 190 (2009) 247.

[6] M.Aglietta et al. (EAS-TOP Collaboration), Astroparticle Physics 21 (2004) 583.

[7] P.Sokolsky et al. (HiRes Collaboration), Nuclear Physics B (Proc. Supp.), 165 (2007) 11.

[8] T.Abu-Zayyad et al., (HiRes-MIA Collaboration) Astrophys.J. 557 (2001) 686-699.

[9] N.M.Budnev et al. (Tunka Collaboration), Proc. 30th ICRC, Merida, Yucatan, Mexico, (2007) arXiv: 0801.3037 .

[10] N.M.Budnev et al. (Tunka Collaboration), Proc. 31th ICRC, Lodz, Poland, 1 (2009) 1069. arXiv: 1003.0089.

[11] B. V. Antokhonov et al. (Tunka Collaboration), to be published in Nucl. Phys. B (Proc. Suppl.) 2011.

[12] E.E.Korosteleva et al. Proc. 31th ICRC, Lodz, Poland, 1 (2009) 0492.

[13] EAS-TOP Collaboration and E.E. Korosteleva, L.A. Kuzmichev, V.V. Prosin, Proc. 28th ICRC, Tsukuba, Japan, 1 (2003) 89.

[14] E. E. Korosteleva et al., Nuclear Physics B (Proc. Supp.), 165 (2007) 74.

[15] J.C.Arteafa-Velazquez et al.(KASCADE-Grande Collaboration) Proc. of XVI ISVHECRI, Batavia, USA ( July 2010), arXiv: 1009.4716

[16] A.P.Garyaka et al., Journal of Physics G: Nuclear and Particle Physics, 35, 115201 (2008)

[17] A.Haungs, Nucl. Instr. and Meth. A604 (2009) 632.

[18] O. Kroemer et al., Proc of 31st ICRC Lodz, Poland, 1 (2009) 1232

[19] N.N. Kalmykov et al.Proc. 31th ICRC, Lodz, Poland, 1 (2009) 1073

[20] S.I.Skribykina, Diploma Thesis, MSU, 2008 (in Russian), supervisor N.N.Kalmykov. 